\title{
Successful treatment of epiglottitis with two doses of ceftriaxone
}

\author{
S M Sawyer, P D R Johnson, G G Hogg, C F Robertson, F Oppedisano, S J MacIness, \\ G L Gilbert
}

\begin{abstract}
Epiglottitis in childhood is caused by Haemophilus influenzae type b. The usual antibiotic treatment at the Royal Children's Hospital, Parkville, Victoria is a five day course of chloramphenicol. Increasingly, third generation cephalosporins are being used to treat invasive $H$ influenzae type $b$ infections and preliminary data suggest that they can be used successfully for epiglottitis. In a prospective, randomised trial, the efficacy of a short course (two days) of ceftriaxone was compared with that of five days of chloramphenicol for the treatment of epiglottitis. The ability of these treatment regimens to eradicate $H$ influenzae type $b$ from the throat was also studied. Fifty five children were enrolled over an 18 month period. Epiglottitis was diagnosed clinically and confirmed on inspection of the epiglottis at direct laryngoscopy. Fifty three $(96 \%)$ of 55 patients had $H$ influenzae type $b$ detected from at least one site: $44 / 52 \quad(85 \%)$ from blood cultures, $41 / 47$ (87\%) from throat swab, and $6 / 8(75 \%)$ as $H$ influenzae
\end{abstract} type b urinary antigen. Children were randomised to receive either ceftriaxone $100 \mathrm{mg} / \mathrm{kg}$ intravenously followed by a single dose of $50 \mathrm{mg} / \mathrm{kg} 24$ hours later (28 patients), or chloramphenicol 40 $\mathrm{mg} / \mathrm{kg}$ intravenously, then $25 \mathrm{mg} / \mathrm{kg}$ eight hourly for five days, intravenously then by mouth (27 patients). All household contacts and patients receiving chloramphenicol received rifampicin 20 $\mathrm{mg} / \mathrm{kg}$ daily for four days. Index patients randomised to ceftriaxone were not treated with rifampicin. There was no significant difference in outcome between the two groups with respect to the mean duration of fever, the duration of intubation, or the length of hospital admission. The proportion of patients colonised with $H$ influenzae type b four weeks after discharge was not significantly different between the two groups: ceftriaxone $5 / 22(23 \%)$ versus chloramphenicol and rifampicin $3 / 23(13 \%)$. A short course of ceftriaxone was successful in treating all patients with no significant side effects and no relapses. A short course of ceftriaxone is a safe, efficacious, and economic alternative to the standard treatment in children with epiglottitis.

(Arch Dis Child 1994; 70: 129-132)
Epiglottitis is an acute infection caused by Haemophilus influenzae type $\mathrm{b}$ that can cause life threatening airway obstruction. The annual attack rate for all invasive $H$ influenzae type $b$ disease in the state of Victoria is $58 \cdot 5 / 100000$ children under the age of 5 years, with epiglottitis accounting for $40 \%$ of all invasive disease. ${ }^{1}$ Epiglottitis in Victoria and other Australian states constitutes a higher proportion of invasive $H$ influenzae type $\mathrm{b}$ disease than in many other countries. ${ }^{2}$

Airway management and the use of antibiotics are the most important aspects of treatment. The technique of nasotracheal intubation for the management of children with upper airway obstruction, developed in Australia in the 1960 s, is now widely accepted as the preferred method of maintaining airway patency in epiglottitis. Ninety per cent of children with epiglottitis require intubation but they are not usually ventilated. ${ }^{3}$ There is rapid recovery; patients are generally afebrile and can be extubated within 24 hours of admission, and at this hospital are usually discharged on the third day. ${ }^{3}$

There is less consensus regarding the choice and duration of antibiotic treatment. The choice is influenced by local patterns of drug resistance. In this community, $25 \%$ of blood culture isolates are resistant to penicillin and chloramphenicol resistance is rare (Royal Children's Hospital, unpublished data). At this hospital for many years a five day course of chloramphenicol given by intravenous infusion followed by treatment by mouth has been used. In recent years this has been followed by a four day course of rifampicin to eradicate $H$ influenzae type $\mathrm{b}$ throat carriage. Third generation cephalosporins are increasingly used to treat invasive $H$ influenzae type $\mathrm{b}$ disease in children, but there is little documentation of their use in epiglottitis. A single dose of ceftriaxone $100 \mathrm{mg} / \mathrm{kg}$ each day for five days was effective in a small uncontrolled study of children with epiglottitis. ${ }^{4}$ The rapid response to treatment and the rarity of complications suggests that a shorter course may be as efficacious. The use of rifampicin after treatment with chloramphenicol was shown to eradicate nasopharyngeal carriage, which persists in $34 \%$ of patients after successful treatment of epiglottitis. ${ }^{5}$ It is not known whether cephalosporins reliably eradicate $H$ influenzae type b carriage in epiglottitis.

The objectives of this study were (a) to compare prospectively the efficacy of a five day course of chloramphenicol with that of two doses of ceftriaxone given 24 hours apart in the 
treatment of epiglottitis and (b) to compare the eradication rate of $H$ influenzae type $\mathrm{b}$ in patients with epiglottitis treated with ceftriaxone with that in patients treated with chloramphenicol and rifampicin.

\section{Patients and methods PATIENTS}

All children admitted to the Royal Children's Hospital between May 1991 and December 1992 with the clinical diagnosis of epiglottitis, who required intubation, were eligible for entry to the study. Children were considered to have epiglottitis if they were acutely ill with fever, stridor, drooling, the absence of a harsh cough, and an acutely inflamed epiglottitis at laryngoscopy. Patients were excluded if intravenous antibiotics had been given within 24 hours before admission, or if there was a history of allergy to $\beta$ lactam antibiotics, or if patients were immunosuppressed. Parental consent was obtained for all patients and this study was approved by the Royal Children's Hospital ethics in human research committee.

\section{MICROBIOLOGICAL METHODS}

Bacteriological confirmation was attempted in all patients. Blood culture, throat swab, and urine were collected. A urinary antigen test for $H$ influenzae type $\mathrm{b}$ was performed only if $H$ influenzae type $\mathrm{b}$ was not isolated from blood culture or throat swab. A nasopharyngeal aspirate was taken within 12 hours of admission for the detection of viruses by immunofluorescence and culture. Throat swabs were taken using a standard technique and inoculated onto haemophilus antiserum and chocolate bacitracin agars. ${ }^{6}$ Plates were examined after 24-36 hours of incubation at $35^{\circ} \mathrm{C}$ in $5 \%$ carbon dioxide. Suspicious colonies were subcultured and identified by their growth requirements for $\mathrm{X}$ and $\mathrm{V}$ factors and by slide agglutination with commercial $H$ influenzae type $\mathrm{b}$ antiserum (Wellcome). Isolates of $H$ influenzae type $\mathrm{b}$ were tested for $\beta$ lactamase production with a commercial nitrocefin disc (BBL) and for susceptibility to penicillin, chloramphenicol, ceftriaxone, and rifampicin by a disc diffusion method.

RANDOMISATION AND DRUG DOSAGE

Using a block of four design, patients were randomised before the start of treatment to receive ceftriaxone or chloramphenicol. The dose of ceftriaxone was $100 \mathrm{mg} / \mathrm{kg}$, followed by $50 \mathrm{mg} / \mathrm{kg} 24$ hours later. The dose of chloramphenicol was $40 \mathrm{mg} / \mathrm{kg}$ followed by $25 \mathrm{mg} / \mathrm{kg}$ eight hourly for five days. Chloramphenicol was given intravenously until the patient was able to tolerate fluids by mouth, from which time it was given orally. This was followed by rifampicin $20 \mathrm{mg} / \mathrm{kg}$ daily by mouth for four days. All family members of the two patient groups were given rifampicin. Rifampicin was not given to patients treated with ceftriaxone.

\section{GENERAL MANAGEMENT}

Patients were managed in the intensive care unit with nasopharyngeal intubation and spontaneous respiration without sedation. ${ }^{3}$ Criteria for extubation included the resolution of temperature to $37.5^{\circ} \mathrm{C}$ and an improvement in the child's general appearance. Patients were transferred to the general ward after a four hour period of observation after extubation, and were discharged home once they were afebrile and tolerating fluids by mouth.

\section{OUTCOME CRITERIA}

Outcome was analysed according to (a) duration of fever (time to axillary temperature less than or equal to $37.5^{\circ} \mathrm{C}$ at four consecutive 15 minute intervals), (b) duration of intubation, (c) time until fluids were tolerated by mouth, and (d) length of hospital admission. Follow up patients were reviewed four to six weeks after discharge by a single investigator (SMS). Questions were asked about possible drug reactions (for example skin rash or diarrhoea), other illness, subsequent use of antibiotics, compliance with rifampicin treatment. A throat swab and a nasopharyngeal aspirate were obtained. A four day course of rifampicin was subsequently prescribed if $H$ influenzae type $\mathrm{b}$ was isolated from the follow up throat swab.

\section{STATISTICAL METHODS}

Parametric data were analysed by the unpaired $t$ test. Non-parametric data were analysed by Fisher's exact test or the $\chi^{2}$ test as appropriate. Statistical significance was taken as $\mathrm{p}<0 \cdot 05$.

\section{Results}

During the 18 months of the study 85 children were admitted with the diagnosis of epiglottitis. Thirty were excluded either because of previous parenteral antibiotic treatment ( 24 children) or because intubation was not required (five children). An additional patient was excluded because of aspiration during intubation before transfer to this hospital. Fifty five patients were therefore enrolled. The mean age of those enrolled was 34 months (median 32 months, range 7-68 months), which was not significantly different from that of those excluded. Eight $(15 \%)$ of 55 children were less than 18 months old. The male:female ratio was 1.75 .

Epiglottitis was diagnosed clinically and confirmed at laryngoscopy in all patients. Fifty three of $55(96 \%)$ patients had $H$ influenzae type b detected. $H$ influenzae type b was isolated from $44 / 52(85 \%)$ blood cultures and no other organism was cultured from blood cultures in any patient. Forty one $(87 \%)$ of 47 patients had $H$ influenzae type b isolated from the initial throat swab, and $H$ influenzae type $b$ antigen was detected in the urine of one other patient. Four patients had either negative or unavailable blood, throat, and urine specimens.

Nine $(23 \%)$ of 40 patients had a virus isolated from the initial nasopharyngeal 
Mean (95\% confidence intervals) values for each of four trial endpoints

\begin{tabular}{|c|c|c|c|}
\hline Endpoint (hours) & Ceftriaxone & Chloramphenicol & p Value ${ }^{\star}$ \\
\hline Duration of fever & $8 \cdot 8(7 \cdot 3$ to $10 \cdot 3)$ & $10 \cdot 1(8.9$ to 11.3$)$ & $0 \cdot 17$ \\
\hline Duration of intubation & $15 \cdot 3(13 \cdot 7$ to $17 \cdot 4)$ & $17 \cdot 7(15 \cdot 0$ to $20 \cdot 3)$ & $0 \cdot 18$ \\
\hline Feeding started & $23 \cdot 4(22 \cdot 1$ to $24 \cdot 7)$ & $24.8(23.0$ to $26 \cdot 6)$ & $0 \cdot 18$ \\
\hline Duration of admission & $51 \cdot 0(46 \cdot 7$ to $55 \cdot 3)$ & $49.7(45.0$ to 54.5$)$ & 0.69 \\
\hline
\end{tabular}

${ }^{\star}$ Unpaired $t$ test.

aspirate. Seven were respiratory viruses: three respiratory syncytial virus and four rhinovirus.

Twenty eight patients received ceftriaxone and 27 chloramphenicol. There was no difference in the age or sex ratio between the two groups. Treatment was successful in the two groups, and there was no difference in the duration of fever, duration of intubation, time to start of feeding, or length of hospital admission. Based on these results (table), clinically important differences in the rate of recovery from epiglottitis, defined as a 24 hour reduction in hospital stay and a 12 hour reduction in duration of fever, have been excluded with a power of $99 \%(\alpha=0.05)$. Means and $95 \%$ confidence intervals for the duration of fever and duration of admission were: chloramphenicol duration of fever $10 \cdot 1(8.9$ to 11.3$)$, ceftriaxone duration of fever $8.8(7.3$ to 10.3$)$ hours; chloramphenicol duration of admission 49.7 $(45.0$ to 54.5$)$, ceftriaxone duration of admission $51 \cdot 0$ ( $46 \cdot 7$ to $55 \cdot 3$ ) hours.

One child developed a generalised urticarial rash 18 hours after starting treatment with chloramphenicol and was changed to ceftriaxone with a rapid resolution of urticaria. There were no significant side effects in the group treated with ceftriaxone.

Thirteen (29\%) of $45 \mathrm{H}$ influenzae type b isolates were resistant to penicillin; none was resistant to chloramphenicol, ceftriaxone, or rifampicin.

\section{FOLLOW UP RESULTS}

Follow up was achieved in 53/55 patients $(96 \%)$; in person in $82 \%$ and by telephone in $14 \%$. Specific inquiry at follow up showed that all patients were well, with no relapse, recurrence, or significant side effects attributable to either drug. $H$ influenzae type $\mathrm{b}$ was isolated from $5 / 22(23 \%)$ throat swabs of patients treated with ceftriaxone, and $3 / 23$ $(13 \%)$ patients treated with chloramphenicol and rifampicin (difference not significant). All patients complied with the prescribed rifampicin regimen except for one parent who admitted taking only two of the four recommended doses.

A nasopharyngeal aspirate was collected from 40 patients; a virus was isolated from three (two respiratory: one respiratory syncytial virus and one adenovirus); eight were unsatisfactory specimens. Thus the respiratory viral isolation rate was $2 / 32(6 \%)$.

\section{Discussion}

Chloramphenicol has been used successfully for many years in Australia to treat epiglottitis. There is concern about side effects, however, the development of chloramphenicol resistant $H$ influenzae type $\mathrm{b}$ and, more recently, the proposed withdrawal from the local market of the oral chloramphenicol suspension. Severe side effects are well recognised, but rare; the risk of fatal aplastic anaemia is estimated to be one in $20000 .{ }^{7}$ Chloramphenicol resistance is also uncommon in this community. Despite this long and successful experience, ${ }^{3}$ prescribing patterns for antibiotics are apparently changing as $14 / 24(58 \%)$ patients excluded from this study had been given third generation cephalosporins before admission to this hospital.

Five days has been the standard duration of chloramphenicol treatment of epiglottitis in Australia. There is little but historical precedent to support this. Epiglottitis is an invasive $H$ influenzae type b infection and $85 \%$ of patients in this study had positive blood cultures. Resolution of illness occurs quickly with a rapid defervescence of temperature, a short period of intubation, and a mean length of hospital stay of only 50 hours. H influenzae type $b$ disease at other sites, relapse, or recurrence is rare. These are compelling reasons to reconsider the appropriate duration of treatment for this infection.

Ceftriaxone is a third generation cephalosporin with a long elimination half life, excellent tissue penetration, and proved activity against $H$ influenzae type b. ${ }^{8}$ Knight et al reported the successful treatment of epiglottitis in seven children using a single dose of $100 \mathrm{mg} / \mathrm{kg}$ ceftriaxone once a day for five days. ${ }^{4}$ A five day course of ceftriaxone is an expensive alternative to chloramphenicol and the same group of workers have reported the successful treatment of 11 patients with a single dose of $100 \mathrm{mg} / \mathrm{kg}$ ceftriaxone once a day for three days. ${ }^{9}$ Both were uncontrolled studies.

This study shows that two doses of ceftriaxone were efficacious in the treatment of 28 patients with epiglottitis, with no relapses, recurrences, or side effects. Although this study lacked the power to address the question of efficacy, it was sufficiently powerful to exclude important differences in the rate of recovery from epiglottitis. We did not observe significant differences between patients who received ceftriaxone and those who received chloramphenicol for any of the trial endpoints.

Criticism of the use of cephalosporins in invasive disease has been in part because of the acquisition cost of the drug. The frequency and rate of administration also has a significant effect on total cost, however. Although the acquisition cost of two doses of ceftriaxone is higher than five days of chloramphenicol (A \$56 v A \$35), the overall costs when administration costs such as nursing time and disposables are included ${ }^{10}$ are equivalent for an 'average' $15 \mathrm{~kg}$ child (A\$64 v A $\$ 67$ ). Rifampicin for patients and family members is an additional cost.

Pharyngeal carriage of $H$ influenzae type $b$ is reduced but not reliably eradicated by treatment with chloramphenicol. The American Academy of Pediatrics ${ }^{11}$ and the National 
Health and Medical Research Council (Australia), among other authorities, recommend that, if the contact group includes a child under the age of 4 years, the whole group should receive rifampicin prophylaxis to eradicate throat carriage of $H$ influenzae type b. ${ }^{12}$ A study of rifampicin prophylaxis in patients with $H$ influenzae type $b$ disease at this hospital showed that $34 \%$ of patients treated with chloramphenicol but not given rifampicin had positive throat swabs at follow up, compared with only $7 \%$ in those given rifampicin prophylaxis. In families in which all members received rifampicin, only $3 \%$ of children were colonised with $H$ influenzae type $\mathrm{b}$ at follow up. 5

Despite the significantly increased risk of $H$ influenzae type $\mathrm{b}$ disease among close contacts, recognised disease in close contacts accounts for only $1-2 \%$ of cases, and rifampicin prophylaxis, as recommended, is time consuming and costly. A review of prescribing habits for rifampicin prophylaxis at this institution showed that rifampicin was prescribed correctly in only $59 \%$ of family members including the index case, and was not prescribed in $34 \%$ of families in whom it was indicated according to the current recommendations. ${ }^{5}$

It would be advantageous if the same antibiotic used to treat epiglottitis could also eradicate $H$ influenzae type b carriage. $H$ influenzae type $\mathrm{b}$ was eradicated from the throats of nine of nine patients with epiglottitis treated with a single dose of $100 \mathrm{mg} / \mathrm{kg}$ ceftriaxone each day for three days. ${ }^{9}$ In a small uncontrolled study, two of six day care centre contacts of children with invasive $H$ influenzae type $\mathrm{b}$ disease had positive throat swabs for $H$ influenzae type b after a single dose of $125 \mathrm{mg}$ ceftriaxone was given intramuscularly, and $8 / 13$ remained positive after a single $250 \mathrm{mg}$ dose. ${ }^{13}$ The effect of cephalosporins in eradicating the throat carriage of $H$ influenzae type b has not been systematically addressed, however.

This study shows that ceftriaxone without rifampicin, like chloramphenicol, does not reliably eradicate $H$ influenzae type b from the nasopharynx. A significant difference in $H$ influenzae type b carriage rates after treatment between the two groups cannot be excluded as nearly 2000 patients would be required to demonstrate a $10 \%$ difference. As a result, there is no indication to change existing guidelines for the use of rifampicin.

Little is known about the pathogenesis of epiglottitis or its relation with preceding or concurrent viral infection. Although most children are probably colonised with $H$ influenzae type $\mathrm{b}$ during early childhood, most do not develop invasive disease. ${ }^{14}$ The events leading to transition from colonisation to invasion are unknown, but it is logical to implicate viral coinfection. Respiratory syncytial virus in children is sometimes followed by evidence of bacterial infection, ${ }^{15}$ and there is a clear association between secondary bacterial infections and influenza in adults. ${ }^{1617}$
$H$ influenzae type $\mathrm{b}$ disease, including epiglottitis, has a seasonal incidence in this and other communities that may follow patterns of respiratory illness, ${ }^{518}$ but the evidence of a causal relation is far from conclusive. ${ }^{19}$ In this study, $17 \%$ of patients from whom a nasopharyngeal aspirate was obtained during their acute illness also had a detectable respiratory virus, compared with $7.5 \%$ of patients at follow up (conversely, $83 \%$ of patients did not have virus detected by culture nor immunofluorescence). This may be because viral coinfection either does not play a major part in the pathogenesis of $H$ influenzae type $b$, or because viral infection precedes epiglottitis and is not detectable at presentation.

In conclusion, short term treatment with ceftriaxone was used successfully in 28 patients with epiglottitis. It was simple to administer, well tolerated, cost effective, and overcame the need for drug treatment after discharge. It is an efficacious and safe alternative to five days of treatment with chloramphenicol.

The authors gratefully acknowledge the assistance of the medical and nursing staff of ICU, 8 East and 8 West, Jodi Lilly, the scientific staff of the department of microbiology and infectious scientific staff of the department of microbiology and infectious
disease, the pharmacy department and the department of disease, the pharmacy department and the department of
thoracic medicine, Royal Children's Hospital. Dr Sawyer was thoracic medicine, Royal Children's Hospital. Dr Sawyer was
supported by a Clinical Research Scholarship, Royal Children's Hospital Research Foundation. The study was funded by Roche Pharmaceuticals.

1 Gilbert GL, Clements DA, Broughton SJ. Haemophilus influenza type $b$ infections in Victoria, Australia 1985-1987. Pediatr Infect Dis 1990; 9: 252-7.

2 Sly PD, Landau LI, Wagener JS. Acute epiglottitis in childhood: report of an increased incidence in Victoria. Aust $N$ Z F Med 1984; 14: 131-4.

3 Butt W, Shann F, Walker C, Williams J, Duncan A, Phelan P. Acute epiglottitis: a different approach to management. Crit Care Med 1988; 16: 43-7.

4 Knight GL, Harris MA, Parbari M, O'Callaghan MJ, Masters IB. Single daily dose ceftriaxone therapy in epiglottitis. F Padiatr Child Health 1992; 28: 220-2.

5 Gilbert GL, MacInnes S, Guise I. Rifampicin prophylaxis for throat carriage of Haemophilus influenzae type $b$ in patients with invasive disease and their contacts. $B M \mathcal{F}$ 1991; 302: 1432-5.

6 Michaels R, Stonebraker F, Robbins JB. Use of antiserum agar for detection of Haemophilus influenzae type $b$ in the pharynx. Pediatr Res 1975; 9: 513-6.

7 Kucers A, Bennett NMcK. The use of antibiotics. London: Heinemann, 1987: 779-80.

8 Steele RW. Ceftriaxone: increasing the half-life and activity of third generation cephalosporins. Pediatr Infect Dis 1985; 4: $188-91$.

9 Justo RN, Masters IB. Towards short-course single daily dose. Pediatr Infect Dis 1991; 10: 477.

10 Plumridge RJ. Cost comparison of intravenous antibiotic administration. Med f A Aust 1990; 153: 516-8.

11 American Academy of Pediatrics, Committee on Infectious Disease. Revision of recommendation for use of rifampicin prophylaxis of contacts of patients with Haemophilus influenzae infection. Pediatrics 1984; 74: 301-2.

12 National Health and Medical Research Council (Australia). Report of 100th session. Canberra: Australian Government Publishing Service, 1985.

13 Rowley AH, Chadwick EG, Kabat K, Sroka P, Shulman ST, Yogev R. Failure of a single dose of ceftriaxone to eradicate nasopharyngeal colonization of Haemophilus influenzae type b. F Pediatr 1987; 110: 792-4.

influenzae type b. $\mathcal{F}$ Pediatr $1987 ; 110: 792-4$.
14 Moxon ER. The carrier state: Haemophilus influenzae. $f$ Antimicrob Chemother 1986; 18S(a): 17-24.

15 Korppi M, Leinonen M, Koskela M, Makela PH, Launiala $\mathrm{K}$. Bacterial coinfection in children hospitalised with respiratory syncytial virus infections. Pediatr Infect Dis $\mathcal{f}$ 1989; 8: 687-92.

16 Loosli CG. Influenza and the interaction of viruses and bacteria in respiratory infections. Medicine (Baltimore) 1973; 52: 369-84.

17 Mills EL. Viral infections predisposing to bacterial infections. Ann Rev Med 1984; 35: 469-79.

18 Schlech WH 3rd, Ward JI, Band JD, Hightower A, Fraser DW, Broome CV. Bacterial meningitis in the United States, 1978 through 1981. $¥ A M A$ 1985; 253: 1749-54

19 Rosenthal J, Dagan R, Press J, Sofer S. Differences in the epidemiology of childhood community-acquired bacterial meningitis between two ethnic populations cohabiting in one geographic area. Pediatr Infect Dis $\mathcal{f} 1988$; 7: 630-3. 